\title{
COMPLEX ORBITS OF SOLVABLE GROUPS
}

\author{
DENNIS M. SNOW
}

(Communicated by Clifford J. Earle, Jr.)

\begin{abstract}
The following structure theorems are proved: An orbit of a real solvable Lie group in projective space that is a complex submanifold is isomorphic to $\mathbf{C}^{k} \times\left(\mathbf{C}^{*}\right)^{m} \times \Omega$, where $\Omega$ is an open orbit of a real solvable Lie group in a projective rational variety. Also, any homogeneous space of a complex Lie group that is isomorphic to $\mathbf{C}^{n}$ can be realized as an orbit in some projective space. As a consequence, left-invariant complex structures on real solvable Lie groups are always induced from complex orbits in projective space.
\end{abstract}

In [19] it is shown that an orbit of a complex solvable Lie group in complex projective space $\mathbf{P}^{n}$ must be isomorphic as a manifold to $\mathbf{C}^{k} \times\left(\mathbf{C}^{*}\right)^{m}$. The purpose of this note is to prove a similar theorem for an orbit of a real solvable Lie group which is a complex submanifold of $\mathbf{P}^{n}$, not necessarily locally closed. We call such an orbit a complex solvable orbit. The simplest example is the upper half plane $\mathbf{H}$ in $\mathbf{P}^{1}$. In the classification of 2-dimensional homogeneous surfaces [17] other examples turn up: the two-dimensional ball $\mathbf{B}^{2}, \mathbf{H} \times \mathbf{H}$, $\mathbf{C}^{2} \backslash \mathbf{R}^{2}$, and $\mathbf{P}^{2} \backslash\left(\overline{\mathbf{B}^{2}} \cup A\right)$ where $A$ is a projective line tangent to $\mathbf{B}^{2}$. Of course, any homogeneous bounded domain possesses a transitive solvable group of automorphisms. Moreover there exists a realization of the bounded domain as a convex unbounded domain such that the elements of the solvable group act as affine transformations and, therefore, can be extended to a projective space compactification [6].

Complex solvable orbits in projective space are in particular Kähler manifolds, although the Kähler structure need not be invariant under the group. If the Kähler structure is invariant then the manifold falls under a recently completed classification and must be isomorphic to a direct product of a homogeneous bounded domain, an abelian complex Lie group $\left(\mathbf{C}^{n} / \Gamma\right.$ where $\Gamma$ is a discrete subgroup of $\mathbf{C}^{n}$ ), and a compact homogeneous rational manifold (the quotient of a semisimple complex Lie group by a parabolic subgroup) [5]. The proof of this fundamental structure theorem is long and difficult and is the culmination of the efforts of many mathematicians over the course of three decades, see $[7,4]$ for surveys. The case of solvable groups had already been

Received by the editors June 19, 1989 and, in revised form, January 8, 1990.

1980 Mathematics Subject Classification (1985 Revision). Primary 32M10; Secondary 14L30. 
worked out, [8, 3], and of course, no compact homogeneous rational factor can appear.

We show that a similar result can be obtained, using only elementary techniques, for complex solvable orbits in projective space. As a complex manifold such an orbit is isomorphic to $\mathbf{C}^{k} \times\left(\mathbf{C}^{*}\right)^{m} \times \Omega$ where $\Omega$ is an open orbit of a connected real solvable Lie group in a projective rational variety $Z$. Moreover, the real solvable group action is contained in a complex solvable group action on $Z$ such that the complex orbit containing $\Omega$ is Zariski-dense and isomorphic to $\mathbf{C}^{n}$.

General homogeneous spaces of solvable groups do not have such simple structure, even when the groups are complex. Of course, such manifolds need not even admit a Kähler structure, [13]. There is, however, an interesting fibration of homogeneous spaces of complex solvable Lie groups which do admit a Kähler structure [18]. The so-called holomorphic reduction of such a space is a homogeneous fiber bundle with a Stein base and with fiber an abelian complex Lie group without nonconstant holomorphic functions. By Lie's Theorem, a complex orbit of a solvable group in $\mathbf{P}^{n}$ is contained in some linear $\mathbf{C}^{k} \subset \mathbf{P}^{n}$, not necessarily of the same dimension as the orbit, so that the orbit is holomorphically separable. The example of $\mathbf{C}^{2} \backslash \mathbf{R}^{2}$, which can be realized as a complex solvable orbit in $\mathbf{P}^{3}$, shows that in the case of real solvable groups, holomorphically separable does not imply Stein.

Complex solvable orbits also appear in the classification of invariant complex structures on real even dimensional Lie groups. The case of reductive groups is studied in [20]. In that paper it is mentioned that if $G_{0}$ is a simply-connected real solvable Lie group with an invariant complex structure, then $G_{0}$ is the universal cover of an open $G_{0}$-orbit $\Omega \cong G_{0} / \Gamma \subset \mathbf{C}^{n}$, where $\Gamma$ is discrete (see [20, Theorem 1.3]). In this situation the complexification $G$ of $G_{0}$ acts transitively on $\mathbf{C}^{n}$. In $\S 2$ we show that any homogeneous space $G / H$ of a complex Lie group $G$ that is isomorphic to $\mathbf{C}^{n}$ can be realized as an orbit of $G$ in projective space. In particular, invariant complex structures on real solvable Lie groups $G_{0}$ are always induced from complex solvable orbits $\Omega \cong$ $G_{0} / \Gamma \subset \mathbf{C}^{n}=G / H \subset \mathbf{P}^{N}$.

In the last section, we give two examples: The first is a complex solvable orbit in projective space which is isomorphic to $\mathbf{C}^{n}$ but which has no equivariant compactification to any projective variety; the second is a homogeneous space of a complex solvable group $G$ which is isomorphic to $\mathbf{C}^{2} \times \mathbf{C}^{*}$ but which cannot be realized as a $G$-orbit in projective space.

\section{MANifold STRUCTURE OF COMPLEX SOlVABLE ORBITS}

We first prove a lemma about open complex orbits of real Lie groups.

1.1. Lemma. Let $G_{0}$ be a connected real Lie subgroup of a complex Lie group $A$. Assume $A$ acts holomorphically on a complex space $X$ and there exists $a$ $G_{0}$-orbit, $G_{0} \cdot x, x \in X$, that is a complex submanifold of $X$, not necessarily 
closed. Let $G$ be the smallest complex subgroup of $A$ that contains $G_{0}$. Then $G_{0} \cdot x$ is open in $G \cdot x$.

Proof. Let $\mathscr{A}$ be the Lie algebra of $A$ ard let $\phi: \mathscr{A} \rightarrow H^{0}\left(X, T_{X}\right)$ denote the natural homomorphism of complex Lie algebras. Define $\mathscr{J}=\left\{\xi \in \mathscr{A} \mid \phi(\xi)_{y} \in\right.$ $\left.T_{y}\left(G_{0} \cdot x\right), y \in G_{0} \cdot x\right\}$. Since $G_{0} \cdot x$ is a complex submanifold of $X, \mathscr{J}$ is a complex subalgebra of $\mathscr{A}$. Let $J$ denote the associated connected complex subgroup of $A$. Notice that $G_{0} \subset J$ and $T_{x}\left(G_{0} \cdot x\right)=T_{x}(J \cdot x)$. Therefore, $G_{0} \cdot x$ is open in $J \cdot x$. Now suppose $G$ is the smallest complex subgroup of $A$ containing $G_{0}$. Then $G \subset J$ and $G_{0} \cdot x \subset G \cdot x \subset J \cdot x$ so that $G_{0} \cdot x$ is open in $G \cdot x$.

The main structure theorem concerning complex orbits in projective space and its proof follows.

1.2. Theorem. Let $G_{0}$ be a connected solvable Lie group acting as a group of holomorphic automorphisms of some projective space $\mathbf{P}^{N}$. Assume there is a point $x \in \mathbf{P}^{N}$ such that the orbit $X=G_{0} \cdot x$ is a complex submanifold of $\mathbf{P}^{N}$, not necessarily locally closed. Then $X$ is isomorphic as a complex manifold to $\mathbf{C}^{k} \times\left(\mathbf{C}^{*}\right)^{m} \times \Omega$ where $\Omega=J_{0} \cdot x$ is an open orbit of a connected real solvable Lie group $J_{0}$ in a projective rational variety $Z$. Moreover, $J_{0}$ is contained in a complex solvable group $J$ acting holomorphically on $Z$ such that $J \cdot x$ is Zariskidense and isomorphic to $\mathbf{C}^{n}$. The commutator $G^{\prime}$ of $G$ acts algebraically on $Z$ and $G^{\prime} \cdot x=J \cdot x$.

Proof. Lei $G$ be the complex subgroup guaranteed by Lemma 1.1 -here, $A=$ Aut $\mathbf{P}^{N}$-such that $G_{0} \cdot x$ is open in $G \cdot x$. Since $G$ is the smallest complex group containing $G_{0}$ it clearly must also be solvable. As mentioned above, the orbit $G \cdot x$ is isomorphic as a complex manifold to $\mathbf{C}^{k} \times\left(\mathbf{C}^{*}\right)^{m}$, see [19, pp. 209-210].

Let $H$ be the isotropy of the point $x$ so that $G \cdot x \cong G / H$ and $X=$ $G_{0} \cdot x \cong G_{0} / H_{0}$ where $H_{0}=H \cap G_{0}$. According to [19, Lemma 3.2], $H$ is a quasi-algebraic subgroup of $G$. This means that there is some holomorphic representation $\rho$ of $G$ such that $H=\rho^{-1}\left(\rho(H)^{*} \cap \rho(G)\right)$ where $\rho(H)^{*}$ is the algebraic closure of $\rho(H)$. To describe the open subset $X=G_{0} / H_{0}$ of $G / H$, we consider the orbits of the commutator $G^{\prime}$. It is well known that the commutator of a complex linear group is algebraic, and in the case of solvable groups, the commutator is also unipotent [2]. Hence the orbits of $G^{\prime}$ in $G / H$ are all closed in $G / H$ and isomorphic to Euclidean space $\mathbf{C}^{n}$. These orbits are even locally closed in $\mathbf{P}^{n}$. Since $G^{\prime}$ is normal in $G$ its orbits form an equivariant foliation of $G / H$ and the map to the orbit space is an equivariant fibration $\pi: G / H \rightarrow G / J$ with $J=G^{\prime} H$ and fiber $J / H=G^{\prime} / G^{\prime} \cap H \cong$ $\mathrm{C}^{n}$. Restricting this fibration to the open subset $G_{0} / H_{0}$ we obtain the $G_{0^{-}}$ equivariant fibration $\pi_{0}: G_{0} / H_{0} \rightarrow G_{0} / J_{0}$ where $J_{0}=J \cap G_{0}$.

Notice that the commutators of $G, G_{0}$ are contained in $J, J_{0}$, respectively, so that both $G_{0} / J_{0}$ and $G / J$ are abelian groups with $G_{0} / J_{0}$ a subgroup of 
$G / J$. Now, connected abelian Lie groups are generated by any open subset of the identity, and since $G_{0} / J_{0}$ is open in $G / J$, it must equal $G / J$. Moreover, it is straightforward to verify that $J=G^{\prime} H$ is a quasi-algebraic subgroup of $G$ : Let $\rho$ be the above representation. Note that $\rho\left(G^{\prime} H\right)^{*}$ must be contained in the algebraic group $\rho\left(G^{\prime}\right) \rho(H)^{*}$. Hence,

$$
\rho^{-1}\left(\rho\left(G^{\prime} H\right)^{*} \cap \rho(G)\right) \subset G^{\prime} \cdot \rho^{-1}\left(\rho(H)^{*} \cap \rho(G)\right)=G^{\prime} H
$$

showing that $G^{\prime} H$ is quasi-algebraic. Therefore, $G_{0} / J_{0}=G / J \cong A$ where $A=\mathbf{C}^{r} \times\left(\mathbf{C}^{*}\right)^{s}$ [19, Theorem 3.3]. The structure group $G^{\prime}$ of the bundle $\pi$ is contractible so the bundle is topologically trivial. Since the base is a Stein manifold, the bundle must also be holomorphically trivial [9], $G / H \cong A \times \mathbf{C}^{n}$.

We now show that the open $G_{0}$ orbit in $G / H$ is of the form $A \times \Omega$ where $\Omega$ is a connected open orbit of $J_{0}$ in $\mathbf{C}^{n}$. If $\phi: G / H \rightarrow A \times \mathbf{C}^{n}$ denotes an equivariant isomorphism and $z=p r_{2} \circ \phi(x)$ is a fixed point of $H$ in $\mathrm{C}^{n} \cong J / H$, then $S:=\phi^{-1}(A \times\{z\})$ is a 'slice' of $H$-fixed points for the action of $J$ in $G / H$, i.e., every $J$ orbit meets $S$ in exactly one point. A trivialization $\mu: S \times J / H \rightarrow G / H$ can be realized explicitly as $(p, j H) \rightarrow j \cdot p$ where $p \in S$ and $j \in J$. There is some neighborhood $U$ of $x$ in $S$ that is contained in $G_{0} \cdot x$. Then the holomorphic map $\mu$ restricted to the open subset $U \times J_{0} / H_{0}$ has image contained in $G_{0} \cdot x$. In fact, $\mu\left(U \times J_{0} / H_{0}\right)=\pi_{0}^{-1} \pi_{0}(U)$ which shows by homogeneity that the bundle $\pi_{0}$ is holomorphically locally trivial. The bundle is certainly topologically trivial-one can deform the slice $S$ to lie entirely in $G_{0} \cdot x$, for example-so we conclude as above that $\pi_{0}$ is also holomorphically trivial.

Finally, since $\Omega$ is connected, we may replace $J_{0}$ with its connected component, and, since $G^{\prime}$ acts algebraically on $\mathbf{P}^{N}$, its orbit $G^{\prime} \cdot x=J \cdot x \cong \mathbf{C}^{n}$ is locally closed and compactifies equivariantly to a projective rational variety $Z$.

\section{HOMOGENEOUS SPACES ISOMORPHIC TO $\mathbf{C}^{n}$}

If a homogeneous space $G / H$ of a complex Lie group $G$ is isomorphic to $\mathbf{C}^{n}$, then it is not hard to show that the solvable radical of $G$ acts transitively on $G / H,[12,1]$. The problem of realizing $G / H$ as an orbit in projective space then reduces to the case of solvable groups.

2.1. Theorem. Let $G$ be a connected complex Lie group and $H$ a closed complex subgroup such that $G / H$ is isomorphic as a complex manifold to $\mathbf{C}^{n}$. Then there is a holomorphic action of $G$ on some projective space $\mathbf{P}^{N}$ such that $H$ is the isotropy subgroup in $G$ of some point $x \in \mathbf{P}^{N}$ and hence $G / H$ is isomorphic to the complex orbit $G \cdot x \subset \mathbf{P}^{N}$.

Proof. To prove the Theorem we must show that $H$ is quasi-algebraic in $G$ (see [19, Lemma 3.2]). 
By passing to the universal cover of $G$, we may assume $G$ is simply-connected. Since $G / H \cong \mathbf{C}^{n}, H$ is also connected and simply-connected. Let $M \cdot U$ be a Levi decomposition of $G$ where $M$ is semisimple and $U$ is simply-connected and solvable, and let $H=M \cdot V$ be a similar decomposition for $H$. We may assume the semisimple factor for $H$ is all of $M$ because the quotient $G / H$ is homotopically trivial. In fact, one can show that $U$ acts transitively on $\mathbf{C}^{n}$ (see [12, p. 174]). In particular, $G / H=U / V$.

Since simply-connected solvable complex Lie groups always have faithful holomorphic representations, we may assume that $G$ is a linear complex Lie group [10, Theorem 3.6]. Every linear complex Lie group $G$ has an embedding into a linear algebraic group such that $\widehat{G}=T_{G} \cdot G$ is a semidirect product where $T_{G} \cong\left(\mathbf{C}^{*}\right)^{t}$ is a torus in $\widehat{G}$ normalizing $G,[11,15]$. Thus, to prove that $H$ is quasi-algebraic, it is enough to show that $\widehat{H} \cap G=H$ where $\widehat{H}$ is the algebraic closure of $H$ in $\widehat{G}$. The algebraic closure $\widehat{H}$ must also be of the form $\widehat{H}=T_{H} H$ where $T_{H} \cong\left(\mathbf{C}^{*}\right)^{s}$ is a torus in $\widehat{G}$ normalizing $H$ [14]. In general, this decomposition of $\widehat{H}$ is not a semidirect product. However, since $\widehat{G}=M \cdot \widehat{U}$ and $\widehat{H}=M \cdot \widehat{V}$ where $\widehat{U}=T_{G} \cdot U$ and $\widehat{V}=T_{H} \cdot V$ are the algebraic closures of $U$ and $V$ in $\widehat{G}$, we may assume that $T_{H} \subset T_{G}$. Therefore $T_{H} \cap H \subset T_{G} \cap G=1$ and $\widehat{H}=T_{H} \cdot H$ is a semidirect product also. Now, if $g \in \widehat{H} \cap G$ then we may write $g=t \cdot h \in G$ with $t \in T_{H}$ and $h \in H$. But then $t=g h^{-1} \in T_{H} \cap G=1$. Hence, $\widehat{H} \cap G=H$.

The above theorem is useful in the study of real even dimensional solvable Lie groups $G_{0}$ which admit a complex structure invariant under left multiplication. Of course, such a structure is not necessarily right-invariant unless $G_{0}$ is already a complex Lie group. We shall assume that $G_{0}$ is simply-connected. Then there exists an equivariant holomorphic covering map $\pi: G_{0} \rightarrow \Omega=G_{0} / \Gamma$ where $\Gamma$ is a discrete subgroup of $G_{0}$ and $\Omega$ is an open subset of $\mathbf{C}^{n}$. Moreover, the complexification $G$ of $G_{0}$ acts transitively on this $\mathbf{C}^{n}$ [20, Theorem 1.3]. By the above theorem, this $\mathbf{C}^{n}$ is a $G$-orbit in some projective space and $\Omega$ itself is a complex solvable orbit.

2.2. Corollary. Up to discrete equivariant coverings, every real solvable Lie group with a left-invariant complex structure can be realized as a complex solvable orbit in some projective space.

\section{EXAMPles}

Let us now look at two examples which bring into sharper focus some aspects of the previous theorems.

3.1. A noncompactifiable orbit. The first example is a complex solvable orbit $X=G_{0} \cdot x \subset \mathbf{P}^{N}$ of a real solvable Lie group $G_{0}$ which is isomorphic to $\mathbf{C}^{2}$ but which cannot be equivariantly compactified to any projective variety. 
Let $G$ be the four-dimensional complex solvable group defined as the group of matrices:

$$
\left(\begin{array}{ccc}
e^{a} & 0 & b \\
0 & 1 & a \\
0 & 0 & 1
\end{array}\right) \times\left(\begin{array}{ccc}
e^{c} & 0 & d \\
0 & 1 & c \\
0 & 0 & 1
\end{array}\right) \quad a, b, c, d \in \mathbf{C} .
$$

For simplicity, we shall abbreviate the above matrix by $(a, b, c, d)$. Let $G_{0}=\{(u, v, \bar{u}, \bar{v}) \mid u, v \in \mathbf{C}\}$ be a real form $G_{0}$ of $G$ and let $H$ be the closed complex subgroup $\{(a, b, 0, a) \mid a, b \in \mathbf{C}\}$. Then $X=G / H$ is isomorphic to $\mathbf{C}^{2}$. In fact, the map $\phi: G \rightarrow \mathbf{C}^{2}$ defined by $\phi(a, b, c, d)=(c$, $\left.d-e^{c} a\right)$ is invariant under multiplication by $H$ on the right, the subgroup $S=\{(0,0, c, d) \mid c, d \in \mathbf{C}\}$ forms a slice for the action of $H$, and the restriction of $\phi$ to $S$ is an isomorphism onto $\mathbf{C}^{2}$. It is easy to see that the orbit of $G_{0}$ on $G / H$ is the whole space and that $G_{0} \cap H=1$. Thus, this orbit of $G_{0}$ provides the group $G_{0}$ with a left-invariant complex structure.

The ineffectivity of the action of $G$ on $X$ is the normal subgroup $K=$ $\{(0, b, 0,0) \mid b \in \mathbf{C}\}$. Let $G_{1}$ be the simply-connected solvable complex group $G / K$ and let $H_{1}=H / K$ so that $X=G_{1} / H_{1}$ with $G_{1}$ acting effectively on $X$. Now suppose $X$ has a $G$-equivariant-or equivalently, a $G_{1}$-equivariantcompactification as an open subset of a projective algebraic variety $Z$. Identify $X$ with an appropriate orbit $G_{1} \cdot x$ for some $x \in Z$. Let $A$ be the algebraic group of algebraic automorphisms of $Z$ and let $B$ be the algebraic subgroup of those automorphisms that fix the algebraic subvariety $Z \backslash X$. Finally, let $\bar{G}_{1}$ and $\bar{H}_{1}$ be the algebraic closures of $G_{1}$ and $H_{1}$, respectively, in $A$. Recall that these closures can be expressed as products: $\bar{G}_{1}=T_{1} \cdot G_{1}$ and $\bar{H}_{1}=T_{2} \cdot H_{1}$ where $T_{1}$ and $T_{2}$ are algebraic tori in $\bar{G}_{1}$ [14]. Of course, $G_{1}$ and $\bar{G}_{1}$ are contained in $B$ and $X=G_{1} \cdot x=\bar{G}_{1} \cdot x \cong \mathbf{C}^{2}$. In particular, $\mathbf{C}^{2} \cong G_{1} / H_{1}=$ $\bar{G}_{1} / \bar{H}_{1}$ and it follows that $T_{1}=T_{2}$. Since the action of $\bar{G}_{1}$ on $Z$ is effective, $\bar{H}$ contains no nontrivial normal subgroup of $\bar{G}$ and so $T_{1} \cap Z\left(\bar{G}_{1}\right)=T_{2} \cap$ $Z\left(\bar{G}_{1}\right)=1$. This implies that $\bar{G}_{1}$ is the uniquely determined "reduced split hull" of $G_{1}$ (see [16]).

We can compute the reduced split hull of $G_{1}$ directly. Consider the group of matrices

$$
\left(\begin{array}{ll}
1 & a \\
0 & 1
\end{array}\right) \times\left(\begin{array}{ccc}
\mu & 0 & d \\
0 & 1 & c \\
0 & 0 & 1
\end{array}\right) \quad a, c, d \in \mathbf{C}, \mu \in \mathbf{C}^{*}
$$

which is clearly algebraic and contains $G_{1}$ as a Zariski-dense subgroup in the obvious way. Since the center of this group has no nontrivial semisimple elements, it is isomorphic to the reduced split hull of $G_{1},[16$, Theorem 5]. Therefore, we may identify $\bar{G}_{1}$ with this group. However, from this explicit realization of $\bar{G}_{1}$ we see that $T_{1} \cong \mathrm{C}^{*}$ and $T_{2}=1 \quad\left(H_{1}\right.$ is already algebraically closed in $\bar{G}_{1}$ ). This contradicts $T_{1}=T_{2}$. Hence, there can be no $G$-equivariant compactification of $X$ as an open subset of a projective variety $Z$. 
A more informal way to see why there can be no such equivariant compactifications is to inspect the action of the group $G$ on $G / H \cong \mathbf{C}^{2}$. For $(a, b, c, d) \in G$ and $(z, w) \in \mathbf{C}^{2}$,

$$
\begin{aligned}
(a, b, c, d) \cdot(z, w) & =\phi((a, b, c, d)(0,0, z, w)) \\
& =\phi\left(a, b, z+c, e^{c} w+d\right) \\
& =\left(z+c, e^{c} w+d-e^{c+z} a\right) .
\end{aligned}
$$

Regardless of how we choose coordinates $(z, w)$ in $\mathbf{C}^{2}$, it is clear that the two essentially different types of translations

$$
\begin{gathered}
(z, w) \rightarrow(z, w+d) \\
(z, w) \rightarrow\left(z, w+a e^{z}\right)
\end{gathered}
$$

present in the action of $G$ on $G / H$ cannot both be simultaneously extended to an algebraic action on $\mathbf{C}^{2}$.

3.2. A counterexample. Let $G$ be the solvable complex Lie group obtained as the quotient of the group of $3 \times 3$ unipotent matrices modulo a discrete normal subgroup:

$$
\left\{\left(\begin{array}{lll}
1 & x & z \\
0 & 1 & y \\
0 & 0 & 1
\end{array}\right) \mid x, y, z \in \mathbf{C}\right\} \quad \text { modulo }\left\{\left(\begin{array}{lll}
1 & 0 & n \\
0 & 1 & 0 \\
0 & 0 & 1
\end{array}\right) \mid n \in \mathbf{N}\right\} .
$$

As a complex manifold $G$ is clearly isomorphic to $\mathbf{C}^{2} \times \mathbf{C}^{*}$. However, the commutator $G^{\prime}$ of $G$ contains a compact subgroup which is not possible in a linear complex Lie group [11]. In fact, $G^{\prime}$ is the complexification of this compact subgroup and must be contained in the kernel of any holomorphic representation of $G$. Therefore, $G$ itself can never appear as a $G$-orbit in projective space.

\section{REFERENCES}

1. A. Borel, On affine algebraic homogeneous spaces, Arch. Math. 45 (1985), 74-78.

2. C. Chevalley, Théorie des groupes de Lie, Paris, Hermann, 1968.

3. J. Dorfmeister, Homogeneous Kähler manifolds admitting a transitive solvable group of automorphisms, Ann. Sci. École Norm. Sup. 18 (1985), 143-188.

4. _ The radical conjecture for homogeneous Kähler manifolds, CMS Conf. Proc., vol. 5, Amer. Math. Soc., Providence, RI, 1986, pp. 189-208.

5. J. Dorfmeister and K. Nakajima, The fundamental conjecture for homogeneous Kähler manifolds, Acta Math. 161 (1988), 23-70.

6. S. Gindikin, I. Piatetskii-Shapiro, and E. Vinberg, Classification and canonical realization of complex homogeneous domains, Trans. Moscow Math. Soc. 12 (1963), 404-437.

7. __ Homogeneous Kähler manifolds, in Geometry of Homogeneous Bounded Domains, Centro Int. Math. Estivo 3 (1967), 3-87.

8. S. Gindikin and E. Vinberg, Kähler manifolds admitting a transitive solvable automorphism group, Math. USSR-Sb. 3 (1967), 333-351. 
9. H. Grauert, Analytische Faserungen über holomorph-vollständigen Räumen, Math. Ann. 135 (1958), 263-273.

10. G. Hochschild and G. Mostow, Representations and representative functions of Lie groups. III, Ann. Math. 70 (1959), 85-100.

11. (1964), 869-887.

12. A. Huckleberry and E. Oeljeklaus, Homogeneous spaces from the complex analytic viewpoint, in Manifolds and Lie groups, Progress in Math., vol. 14, Birkhäuser, Boston, MA, 1981, pp. 159-186.

13. J. Loeb, Fonctions plurisousharmoniques sur un groupe de Lie complexe invariantes par une forme réelle, C. R. Acad. Sci. Paris 299 (1984), 663-666.

14. A. Magid, Analytic subgroups of affine algebraic groups, Duke J. Math. 44 (1977), 875-882.

15. __ Faithfully representable analytic groups, in Algebraic Geometry Proceedings, Copenhagen, 1978, Lecture Notes in Math., vol. 732, Springer-Verlag, New York, 1979, pp. 384395.

16. _ Representative functions on simply-connected solvable groups, Amer J. Math. 102 (1980), 303-319.

17. K. Oeljeklaus and W. Richthofer, Homogeneous complex surfaces, Math. Ann. 268 (1984), 273-292.

18. _ On the structure of complex solvmanifolds, J. Differential Geom. 27 (1988), 399-421.

19. D. M. Snow, Stein quotients of connected complex Lie groups, Manuscripta Math. 50 (1985), 185-214.

20. _ Invariant complex structures on reductive Lie groups, J. Reine Angew. Math. 371 (1986), 191-215.

Department of Mathematics, University of Notre Dame, Notre Dame, Indiana 46556 